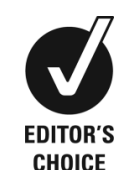

${ }^{1}$ Department of Oral and Maxillofacial Surgery, Government Dental College and Hospital, Ahmedabad Gujarat, India

${ }^{2}$ Department of Oral Pathology and Microbiology, Karnavati School of Dentistry,

Gandhinagar, Gujarat, India ${ }^{3}$ Department of Oral and Maxillofacial Pathology, Kamineni Institute of Dental Sciences, Nalgonda, Telangana, India

\section{Correspondence to} Dr Sumit Bahl, sumitbahl2004@gmail.com

Accepted 21 March 2016
CrossMark

To cite: Anchlia S, Bahl S,
Vyas S, et al. BMJ Case Rep
Published online: [please
include Day Month Year]
doi:10.1136/bcr-2015-
212778

Vyas $\mathrm{S}$, et al. BMJ Case Rep Published online: [please 212778

\title{
Unicystic plexiform ameloblastoma with mural proliferation: a full-blown lesion
}

\author{
Sonal Anchlia, ${ }^{1}$ Sumit Bahl, ${ }^{2}$ Siddharth Vyas, ${ }^{1}$ Godishala Swamy Sugunakar Raju ${ }^{3}$
}

\section{SUMMARY}

Ameloblastoma is the most common aggressive benign odontogenic tumour of the jaws and has received considerable attention due to its frequency, clinical subtypes and high tendency to infiltrate and recur. There are various types of this tumour and confusion still exists among clinicians as to its correct classification.

Multicystic ameloblastoma is the most frequent subtype while unicystic ameloblastoma can be considered a variant of the solid or multicystic subtype. Unicystic ameloblastoma is considered a less aggressive tumour with a variable recurrence rate. However, its frequency is often underestimated. Ameloblastoma is often asymptomatic, presenting as a slowly enlarging facial swelling or an incidental finding on radiograph. It is seen in all age groups but is most commonly diagnosed in the third and fourth decades. We report a case of unusual unicystic ameloblastoma and present its clinical, radiological and full-blown histological changes and treatment modalities, providing new insights into ameloblastomas.

\section{BACKGROUND}

Ameloblastoma is a common odontogenic lesion. We present an uncommon variant of this entity, namely, unicystic ameloblastoma (UA) and describe its histopathological features as a full-blown lesion, such as its follicular, plexiform and mural forms. We wish to share this case with the oral and maxillofacial fraternity and standardise the histopathology to aid in diagnosing these uncommon variants of ameloblastomas.

\section{CASE PRESENTATION}

A 29-year-old man presented with swelling over the lower and middle right third region of the face for 3 months without any history of trauma, fever, toothache, pus discharge and restricted mouth opening. On extraoral examination (figure 1), a single diffuse swelling measuring approximately $4 \times 3.5 \mathrm{~cm}$ was noted on the right lower and middle third region of the face, extending from the corner of the mouth to the angle molar ramus region of the mandible and continuing to the coronoid process. The swelling was non-tender, nonfluctuant and firm and bony hard on palpation.

On intraoral examination, no abnormality was seen except an unerupted 48 (figure 2). Correlating with clinical findings, a provisional diagnosis of ameloblastoma was made; the clinical differential diagnosis is mentioned below:

- Dentigerous cyst

- Keratocystic odontogenic tumour

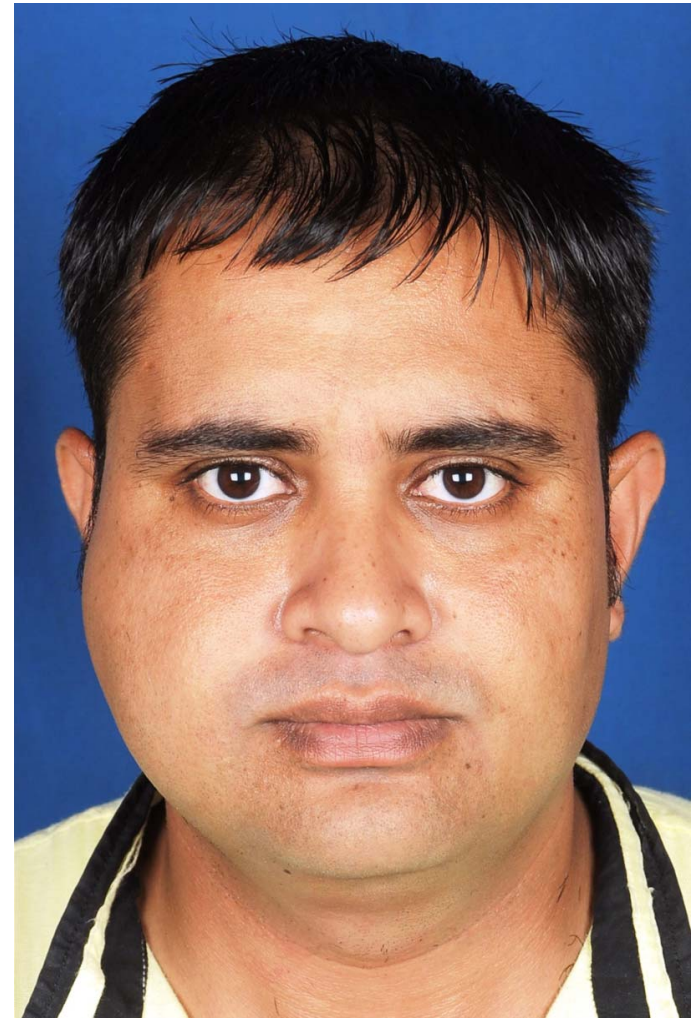

Figure 1 A single huge, non-tender, non-fluctuant, firm and bony hard swelling present on the right lower and middle third region of the face.

The patient was sent for further radiographic and routine haematological investigations. An orthopantomogram (OPG) was taken (figure 3), which showed a well-defined multilocular radiolucency with corticated margins in the region

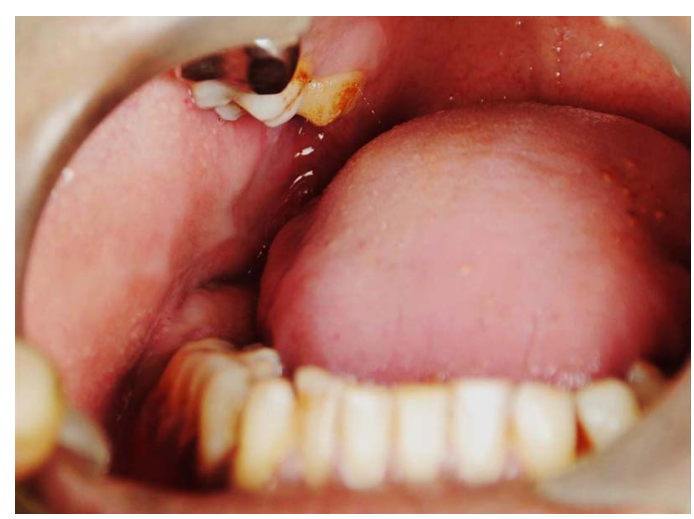

Figure 2 Intraoral examination revealed no abnormalities except an unerupted 48 tooth. 


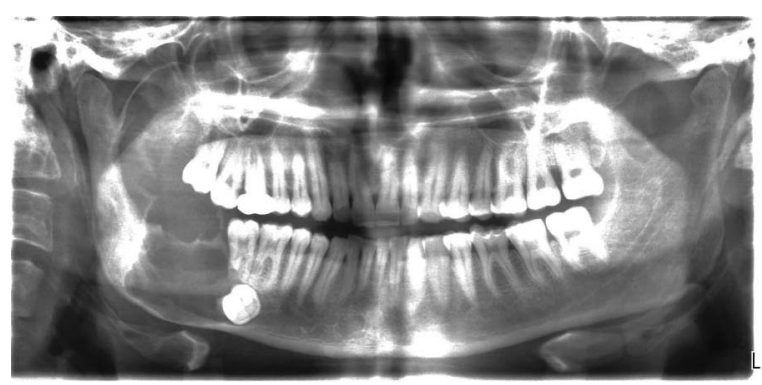

Figure 3 Orthopantomogram revealed a well-defined multilocular radiolucency with corticated margins in the region involving the right molar-angle-ramus region of the mandible with impacted wisdom tooth.

involving the right molar angle ramus region of the mandible and extending to the coronoid process, with an impacted wisdom tooth. In order to confirm the exact location of the lesion, CT scans were taken (figures 4 and 5), which showed a relatively defined multiloculated (80\%), expansile 'soap-bubble' lesion, with well-demarcated borders without matrix calcification, seen on the right side involving the angle-ramus and body of the mandible. The lesion displaced an unerupted permanent tooth 48 inferiorly. The radiological findings were suggestive of ameloblastoma. The patient was advised to undergo an incisional biopsy to confirm the diagnosis of tooth displacement and resorption and soft tissue penetration.

Incisional biopsy was performed; the specimen was fixed, processed and H\&E stained and examined under light microscope. The sections showed (figures 6-9) all the features of unicystic plexiform ameloblastoma with intramural proliferation.

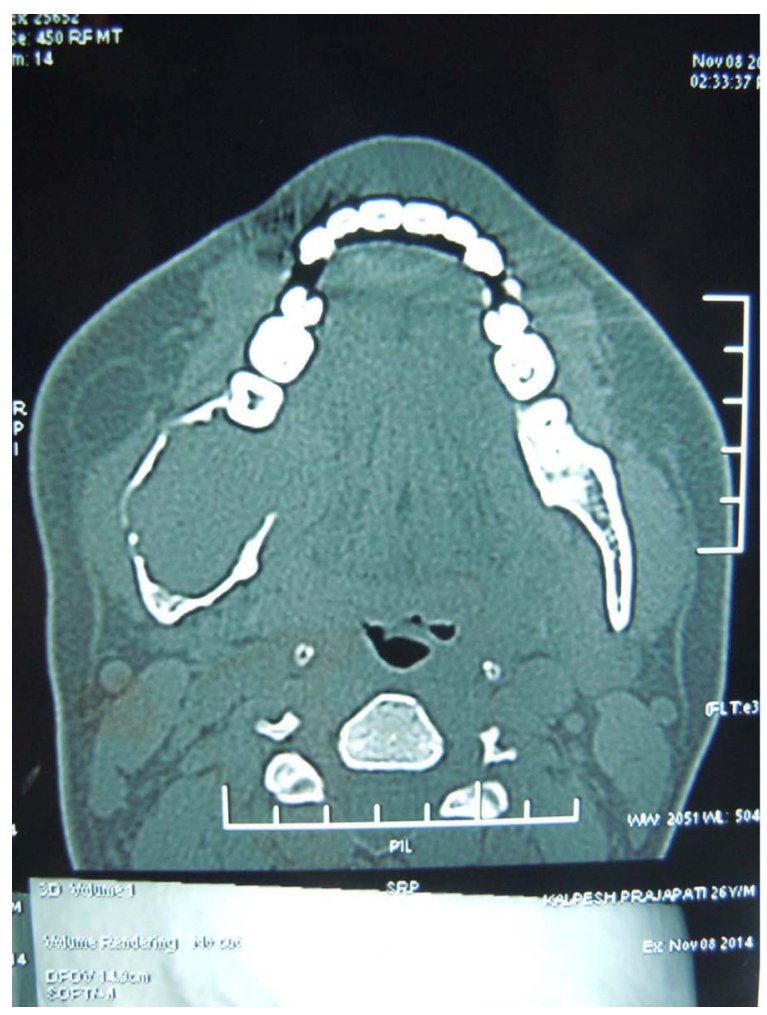

Figure 4 CT axial section revealed radiolucent lesion involving the body angle-ramus region of the mandible with a breach in the cortex of bone.

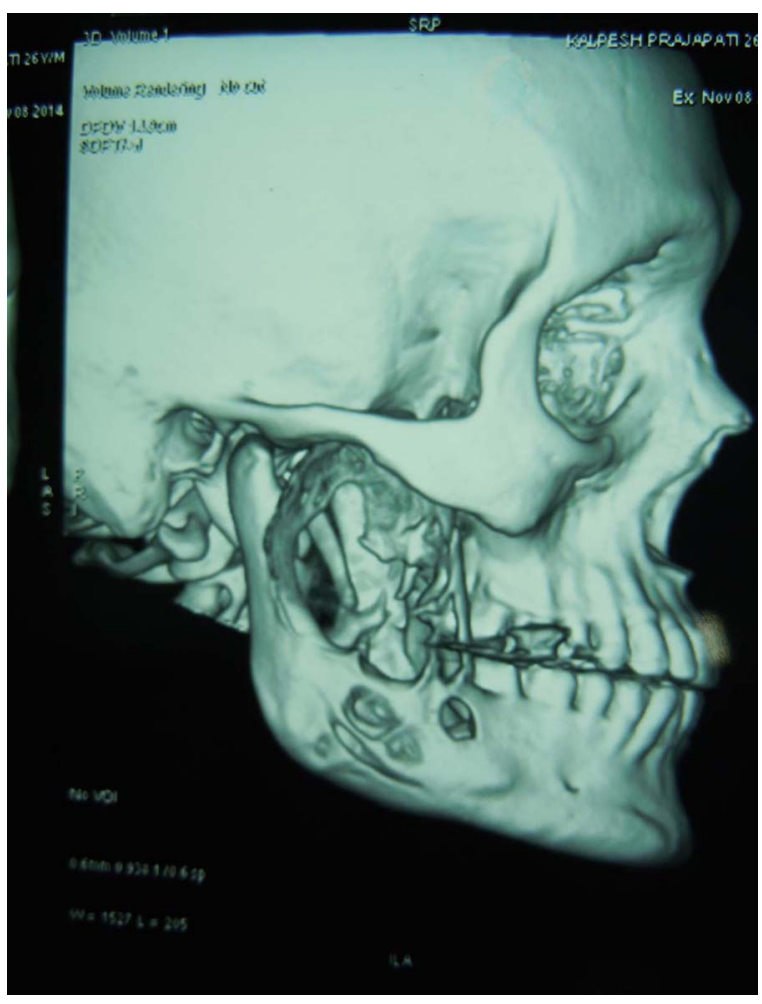

Figure $5 \mathrm{CT}$ (three-dimensional reconstruction) showing lytic areas on the body angle-ramus region of the mandible.

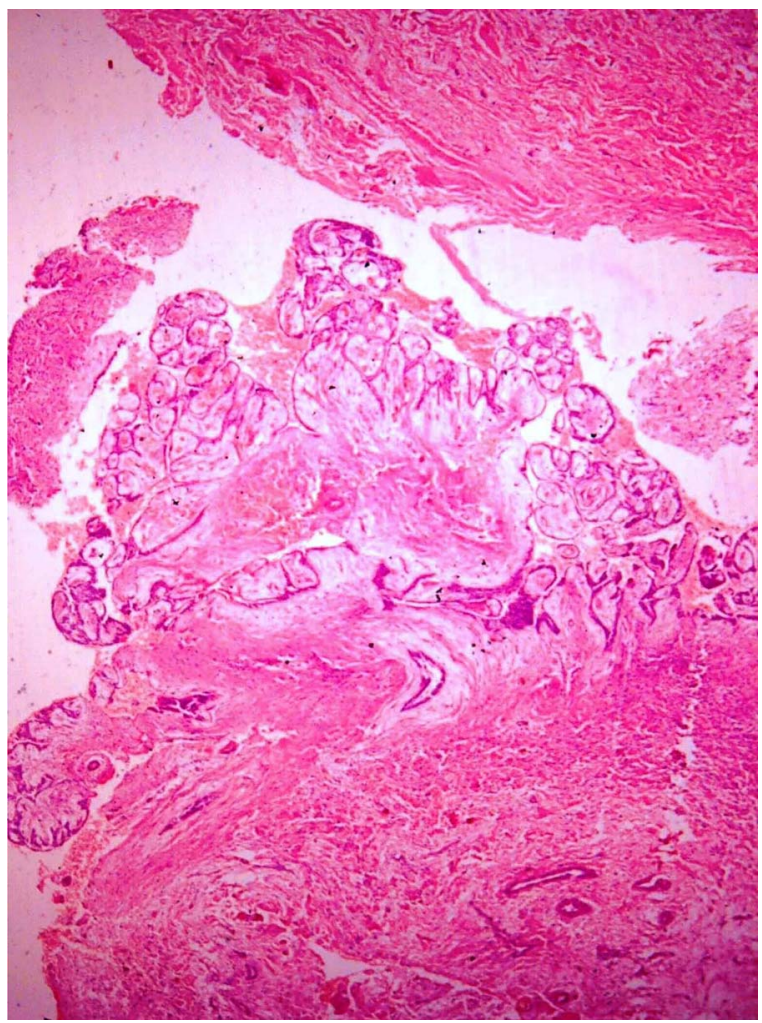

Figure 6 A densely collagenised eosinophilic stromal component and darkly basophilic stained epithelial component in the form of interconnected strands and cords forming a plexus (H\&E stained $4 \times$ photomicrograph). 


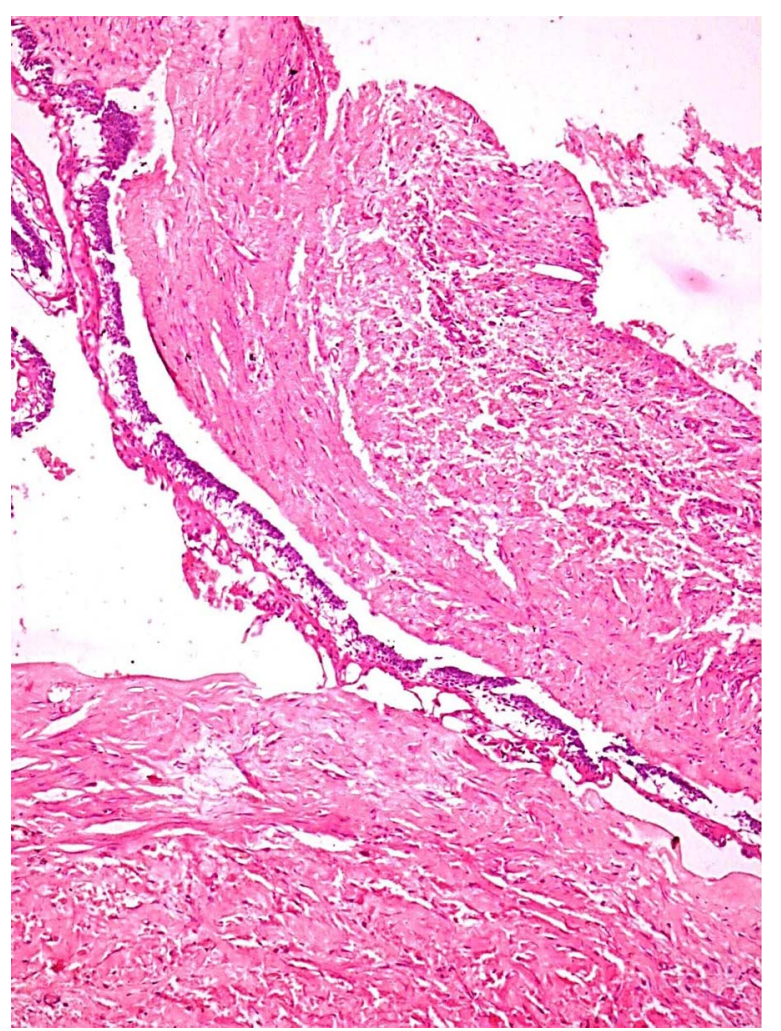

Figure 7 Cystic lumen, cystic lining epithelium and connective tissue wall. Over the epithelium a thin layer of loosely arranged cells, resembling stellate reticulum, is seen and an overlying parakeratinised layer is seen (H\&E stained $4 \times$ photomicrograph).

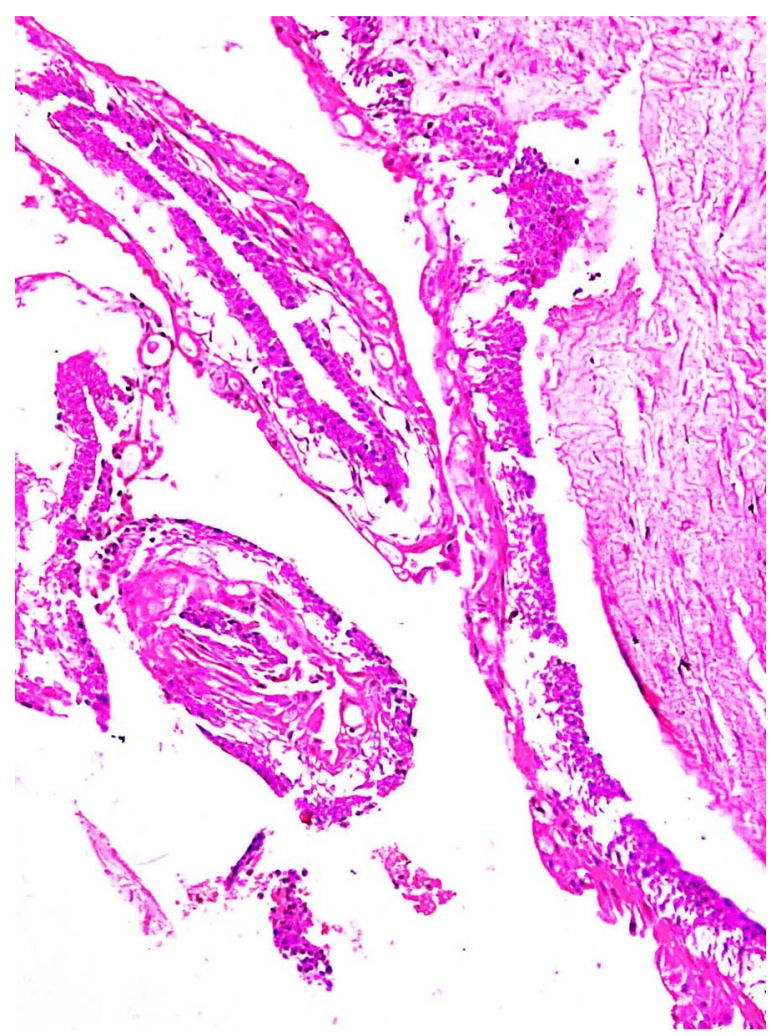

Figure 8 Cystic luminal and intraluminal epithelial proliferation (H\&E stained $20 \times$ photomicrograph).

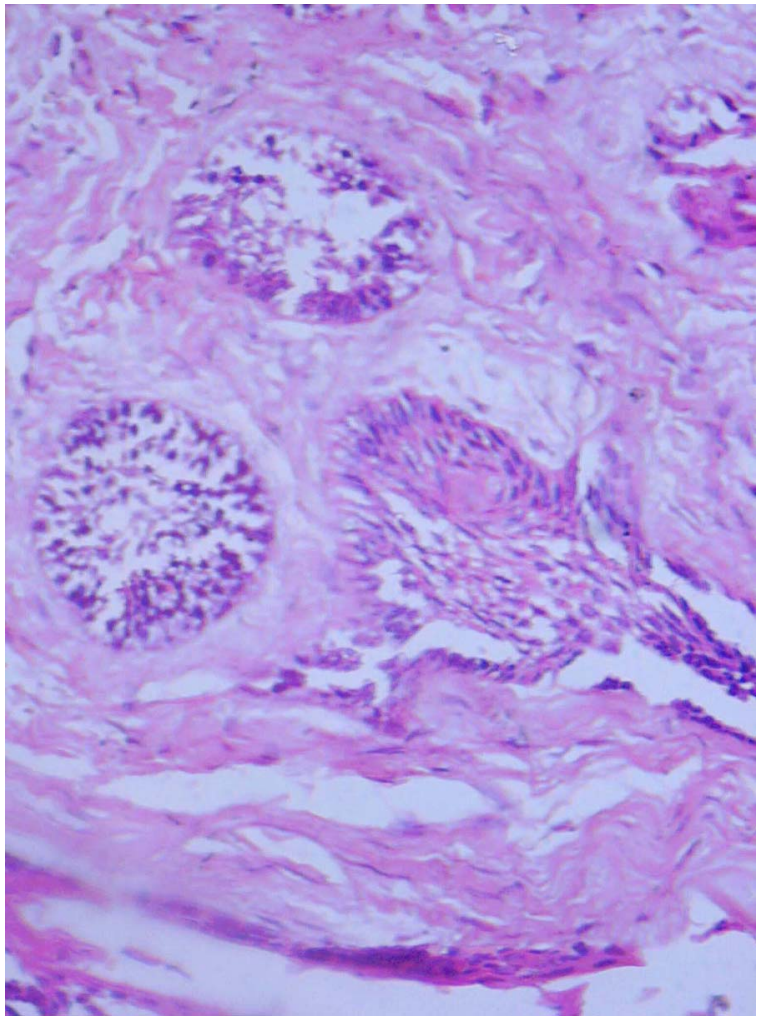

Figure 9 Within the connective tissue wall, evidence of odontogenic epithelial islands, which were in the form of ameloblastic follicles (H\&E stained $40 \times$ photomicrograph).

The histopathological impression was that of UA subgroup: 1.2.3 (luminal, intraluminal and intramural).

All preoperative investigations were conducted and medical fitness was obtained from the patient's physician. A right hemimandibulectomy was performed under general anaesthesia. The pathological specimen from the hemimandibulectomy also revealed unicystic plexiform ameloblastoma with intramural proliferation and postoperative 1, 3 and 6-month and 1-year follow-ups, were uneventful.

\section{INVESTIGATIONS}

\section{Fine-needle aspiration cytology}

- OPG

- CT scan (axial, three-dimensional reconstructions)

- Haematological examination (haemoglobin: $14.5 \mathrm{~g} / \mathrm{dL}$; clotting time: $4 \mathrm{~min}$; bleeding time: $2 \mathrm{~min}$; random blood sugar: $130 \mathrm{mg} / \mathrm{dL}$ )

- Incisional biopsy

Dentigerous cyst and keratocystic odontogenic tumour were ruled out by fine-needle aspiration from the lesional area. In order to better localise the position of pathology, CT radiographic images were taken. To rule out malignancy, incisional biopsy from the lesion area was performed, which showed presence of characteristic features of UA with luminal intraluminal and intramural features.

\section{DIFFERENTIAL DIAGNOSIS}

A working diagnosis of ameloblastoma was given because:

- Ameloblastoma shows equal prevalence around the third to seventh decades of life, as seen in the present case of our 29-year-old man. 
- A painless swelling or expansion with bony hard consistency on the jaw is usual a clinical presentation of ameloblastoma, as seen in the present case.

Clinical differential diagnosis: as an unerupted wisdom tooth 48 was present, a dentigerous cyst or keratocystic odontogenic tumour was suspected, which were ruled out by negative findings in the fine-needle aspiration in our case.

Radiological differential diagnoses as mentioned below were given:

1. Keratocystic odontogenic tumour shows multilocular radiolucency commonly with an associated impacted tooth.

2. Dentigerous cyst is commonly associated with an impacted tooth and has unilocular as well as multilocular radiolucency.

3. Orthokeratinised odontogenic cyst seldom shows multilocular radiolucency associated with an impacted tooth.

\section{TREATMENT}

The decision on the mode of treatment was based on the fact that ameloblastoma showing aggressive features with soft tissue and bony perforation warrants a more aggressive treatment with hemimandibulectomy of the mandible. Postoperative follow-up of 1, 3 and 6 months and 1 year, showed no recurrence and the patient was asymptomatic.

\section{OUTCOME AND FOLLOW-UP}

Nerve paraesthesia persisted for up to 4 months. Postoperatively, the surgical site healed uneventfully with no complications. The patient recovered well with no signs of persistent neurosensory deficit. Follow-up of 1, 3 and 6 months and 1 year, showed no recurrence-the patient remained asymptomatic.

\section{DISCUSSION}

Ameloblastoma is a benign odontogenic tumour of epithelial origin and has received considerable attention due to its frequency, clinical subtypes and high tendency to infiltrate and recur. ${ }^{1}$ Robinson defined ameloblastoma as a tumour that is usually unicentric, non-functional, intermittent in growth, anatomically benign and clinically persistent. ${ }^{2}$ Ameloblastoma may occur centrally within bone, or peripherally, without an intraosseous component in the soft tissue overlying the alveolar ridge. $^{3}$

Intraosseous lesions are of two types: (A) solid/conventional/ multicystic; and (B) unicystic. ${ }^{45}$

Unicystic ameloblastoma as a distinct entity was first recognised by Robinson and Martinez, in $1977 .{ }^{6}$

The growth pattern of UA is seen in approximately $6 \%$ of ameloblastomas. It tends to occur in a younger population compared with the patient population having conventional ameloblastoma. ${ }^{7}$ A high percentage of these lesions are associated with an impacted tooth and the most commonly cited provisional diagnosis is dentigerous cyst. Unicystic ameloblastoma grows in a cystic pattern and has better prognosis and reduced incidence of recurrence compared with conventional ameloblastoma. $^{8} 9$

Most authorities consider ameloblastomas to be of varied origin, although the stimulus initiating the process is unknown. Thus the tumour conceivably may be derived from:

- Cell rests of an enamel organ, either remnants of dental lamina or remnants of Hertwig's sheath, or epithelial rests of Malassez.

- Epithelium of odontogenic cysts, particularly the dentigerous cyst and odontoma.

- Disturbances of a developing enamel organ.
- Basal cells of the surface epithelium of the jaws.

- Heterotopic epithelium in other parts of the body, especially the pituitary gland. ${ }^{6}$

Pathogenic mechanisms proposed for the evolution of UA: ${ }^{10}$

1. Reduced enamel epithelium associated with a developing tooth undergoes ameloblastic transformation with subsequent cystic development.

2. Ameloblastomas arise in dentigerous or other types of odontogenic cyst in which the neoplastic ameloblastic epithelium is preceded temporarily by a non-neoplastic stratified squamous epithelial lining.

3. A solid ameloblastoma undergoes cystic degeneration of ameloblastic islands with subsequent fusion of multiple microcysts and develops into a unicystic lesion.

Unicystic ameloblastoma is a rare type of ameloblastoma, accounting for about $6 \%$ of all ameloblastomas and is most often seen in young patients with $50 \%$ of such tumours diagnosed during the second decade of life. ${ }^{11}$ Compared to multicystic ameloblastoma, UA occurs more commonly at a younger age, in contrast to our case. It most commonly occurs in the posterior mandible, followed by occurrence in the parasymphysis region, anterior maxilla and posterior maxilla. The age is considerably lower and ranges from 19-27 years (Riechart and Philipsen). In our case, the lesion occurred in the body-angle-ramus region of the mandible in a 29 -year-old man.

UA is usually asymptomatic, although a large tumour may cause painless swelling of the jaws with facial asymmetry. Small lesions are sometimes discovered more on radiographic screening examinations or as a result of local effects such as tooth mobility, occlusal alterations and failure of eruption of teeth produced by the tumour. Mucosal ulceration is rare, but may be caused by continued growth of the tumour. Although the histology suggests that UA's follow a biologically low-grade course, they may often clinically behave as biologically aggressive tumours. $^{11}$

There are numerous immunohistochemical studies showing ameloblastic epithelium expressing proliferative cellular nuclear antigen, $\mathrm{Ki} 67$, epidermal growth factor receptor, P53 antibodies indicating a large or huge size attained by the ameloblastoma, ${ }^{12-14}$ and its stromal component showing expression of matrix metalloproteinase-2 (MMP-2), matrix metallopeptidase-9 (MMP-9), receptor activator of nuclear factor $\kappa$ (RANK), RANK ligand (RANKL), OPG and parathyroid hormone-related protein (PTHrp) antibodies, which connotes aggressive bone resorption behaviour shown by ameloblastomas. ${ }^{15-17}$

As UA grows predominantly as a cystic lesion, histopathologically it has a cystic lumen, cystic lining epithelium and cystic wall. The basal cells of lining epithelium are composed of tall columnar cells displaying hyperchromatic, palisaded nuclei and subnuclear vaculisation between basement membrane and a nucleus, which is according to Vickers and Gorlin criteria' given in 1970 for diagnosing ameloblasts. The mural extension into the cystic wall is a frequently seen feature and the term mural UA is used when the thickened lining (either plexiform or follicular) penetrates the adjacent cystic wall (Philipsen et al). ${ }^{11}$

According to Ackermann et al, ${ }^{9}$ UA is subgrouped, as mentioned in table 1 below.

The present case was considered to be a UA subgroup (1.2.3).

The definitive diagnosis of UA can only be carried out by histological examination of the entire lesion and cannot be predicted preoperatively on clinical or radiographic grounds. As preoperative incisional biopsy is not representative of the entire lesion, it may result in an incorrect classification. ${ }^{6}$ 
Table 1 Histological subgrouping of UA by Ackermann et al ${ }^{9}$

\begin{tabular}{ll}
\hline Subgroups & Interpretation \\
\hline 1 & Luminal UA \\
1.2 & Luminal and intraluminal UA \\
1.2 .3 & Luminal, intraluminal and intramural UA \\
1.3 & Luminal and intramural UA \\
\hline UA, unicystic ameloblastoma. &
\end{tabular}

Most of the reported cases of mural proliferation have been of either a plexiform or follicular pattern, or a mixture of the two. However, though rare, we found a combination pattern of follicular, unicystic and plexiform elements. The intramural picture of the present case demonstrates a rare mixture of all these various patterns in a single lesion, as reported in previous published reports. ${ }^{4}$ The radiographic features of the present case showed a typical soap bubble multilocular appearance seen commonly in conventional ameloblastoma, unlike that seen in unicystic radiolucency.

The types of treatment for ameloblastoma include radical or conservative excision. In radical surgical excision, the bone is resected with a 1-2 cm safety margin of macroscopically healthy bone. In conservative excision, the tumour is removed, without safety margin, by enucleation, curettage and curettage followed by cryotherapy with liquid nitrogen; cryosurgery acts as a complementary therapeutic modality, causing cell death in the treated area. ${ }^{15}$ Simple enucleation and curettage have shown a larger number of recurrences than marginal and segmental resections. Hemimandibulectomy is the treatment option in advanced cases of ameloblastoma, particularly when there is involvement of the base of the mandible and condylar process. $^{15} 16$ Radiotherapy has little use in the treatment of ameloblastomas since this tumour is radioresistant, apart from common disadvantages such as osteoradionecrosis and malignant transformation. However, radiotherapy may be useful in the treatment of inoperable cases, because of extensive invasion of neighbouring structures, such as the skull base, particularly in tumours located in the posterior upper jaw. ${ }^{15}$

The recurrence rate for UAs is not zero but is reported to range from $10.7 \%$ to almost $25 \%$. This rate is much lower than the reported recurrence rate for conventional ameloblastoma that are treated only by enucleation or curettage. Regardless of the jaw involved, once an ameloblastoma has recurred, retreatment becomes more challenging. Typically, radical retreatment is performed. In the mandible, this approach has proved to be successful in approximately $80 \%$ of cases. Retreatment of maxillary lesions is more difficult, however. ${ }^{8}$

The average duration of follow-up for ameloblastomas in previous reports was 5 years after the surgical treatment. A recurrence rate of $53 \%$ was found during the first 5 years. ${ }^{4}$ In the present case, no recurrence was observed at 1-year follow-up.

\section{Patient's perspective}

I came to hospital because of swelling in my right middle and lower part of my face for 3 months. Now, after being operated on, I am alright and the swelling has completely subsided.

\section{Learning points}

- Unicystic ameloblastoma, a type of ameloblastoma, also presents with a variety of radiological and histopathological features.

- Ameloblastoma is only diagnosed based on the presence or absence of core histopathological features or Vickers and Gorlin criteria-the nature of the background stroma of the tumour, growth pattern of the epithelium that makes up the tumour, staining pattern of the neoplastic cells, cellular morphology and nuclear orientation.

- This case also highlights the importance of careful examination, radiographic evaluation, detailed observation of the entire biopsy specimen and the usefulness of deeper sections in the diagnosis of unicystic ameloblastoma and ideal and meticulous treatment so as to prevent recurrence.

- Patients with unicystic ameloblastoma have a strong propensity of recurrence and long term follow-up for early recurrence is always warranted.

Acknowledgements The authors would like to thank Dr Swapan Goswami (MD, Pathology, Professor in SBKS Medical College, Piparia, Vadodara, India), Dr Rashmi Bhavasar (Professor, Department of Oral and Maxillofacial Pathology, KM Shah Dental College, Vadodara, India, Dr Bipil Sadhwani (Tutor, Department of Oral and Maxillofacial Surgery, Government Dental College and Hospital, Ahmedabad, Gujarat, India) and Dr Arvind Agarwal (MD, Radiologist, Delhi State Cancer Hospital, New Delhi, India), for their technical support to this case report.

Competing interests None declared.

Patient consent Obtained.

Provenance and peer review Not commissioned; externally peer reviewed.

\section{REFERENCES}

1 Covani U, Barone A. Piezosurgical treatment of unicystic ameloblastoma. J Periodontol 2007;78:1342-7.

2 Rajendran R. Cysts and tumours of odontogenic origin. SHAFER HINE LEVY. Shafers Textbook of oral pathology. 5th edn. Noida: Elsevier publication, 2006:381.

3 Gabane M, Kulkarni M, Mahajan A. Unicystic ameloblastoma of mandible: a case report. Indian J Stomatol 2011;2:273-6.

4 Mahadesh J, Rayapati DK, Maligi PM, et al. Unicystic ameloblastoma with diverse mural proliferation- a hybrid lesion. Imaging Sci Dent 2011; 41:29-33.

5 Kessler HP, Schwartz-Dabney C, Ellis E III. Recurrent left mandibular enlargement. J Contemp Dent Pract 2003;3:127-37.

6 Kumar KR, George GB, Padiyath S, et al. Mural unicystic Ameloblastoma Crossing the Midline: a rare case report. Int J odontostomat 2002;6:97-103.

7 Li TJ, Wu YT, Yu SF, et al. Unicystic ameloblastoma. A clinicopathological study of 33 Chinese patients. Am J Surg Pathol 2000;24:1385-92.

8 Hong J, Yun PY, Chung IH, et al. Long-term follow up on recurrence of 305 ameloblastoma cases. Int J Oral Maxillofac Surg 2007;36:283-8.

9 Ackermann GL, Altini M, Shear M. The unicystic ameloblastoma: a clinicopathological study of 57 cases. J Oral Pathol 1988;17:541-6.

10 Gardener DG, Corio RL. Plexiform unicystic ameloblastoma. A variant of ameloblastoma with a low recurrence rate after enucleation. Cancer 1984;53:1730-5.

11 Kessler HP. Intraoosseous ameloblastoma. Oral Maxillofacial Surg Clin N Am 2004;16:309-22.

12 Hegab A, Shuman M, El-Akher MA, et al. Ki-67 immunohistochemical expression in mandibular ameloblastoma: a prognostic indicator for local recurrence. Open J Stomatol 2013;3:520-6

13 Abdel-Aziz A, Amin MM. EGFR, CD10 and proliferation marker Ki67 expression in ameloblastoma: possible role in local recurrence. Diagn Pathol 2012;7:14 


\section{Unusual presentation of more common disease/injury}

14 Salehinejad J, Zare-Mahmoodabadi R, Saghafi S, et al. Immunohistochemical detection of p53 and PCNA in ameloblastoma and adenomatoid odontogenic tumor. J Oral Sci 2011;53:213-17.

15 Costa H. Free Flaps for Mandible reconstrution. [Doctoral Thesis], University of Porto, December 1995.
16 Costa H. Mandible de Reconstruction- Algorithm for Flap Selection. Proceedings Ipras Congress; New Delli, India, Novembro 2009.

17 Abdelsayed RA, Vartanian RK, Smith KK, et al. Parathyroid hormone-related protein (PTHrP) expression in ameloblastoma. Oral Surg Oral Med Oral Pathol Oral Radiol Endod 2004;97:208-19.

Copyright 2016 BMJ Publishing Group. All rights reserved. For permission to reuse any of this content visit

http://group.bmj.com/group/rights-licensing/permissions.

BMJ Case Report Fellows may re-use this article for personal use and teaching without any further permission.

Become a Fellow of BMJ Case Reports today and you can:

- Submit as many cases as you like

- Enjoy fast sympathetic peer review and rapid publication of accepted articles

- Access all the published articles

- Re-use any of the published material for personal use and teaching without further permission

For information on Institutional Fellowships contact consortiasales@bmjgroup.com

Visit casereports.bmj.com for more articles like this and to become a Fellow 